\title{
Atmospheric Effects on Microphone Array Analysis of Aircraft Vortex Sound
}

\author{
Hadi Wassaf* \\ USDOT John A. Volpe National Transportation Systems Center, Cambridge, MA 02142 \\ Jay C. Hardin ${ }^{\dagger}$ \\ L3 Communications, Billerica, MA 01821 \\ and \\ Frank Y. Wang* \\ USDOT John A. Volpe National Transportation Systems Center, Cambridge, MA 02142
}

\begin{abstract}
This paper provides the basis of a comprehensive analysis of vortex sound propagation through the atmosphere in order to assess real atmospheric effects on acoustic array processing. Such effects may impact vortex localization accuracy and detection probability over longer distances. The paper shows that propagation through the atmosphere can significantly alter the propagation time of the rays from the source to the receiver from that which would be experienced in an ambient atmosphere. More importantly, it can also change the delay time between microphones on which the array processing is based. Implications of these results on the design and resolution of microphone arrays are discussed.
\end{abstract}

\section{Introduction}

$\mathrm{P}$ hased arrays of microphones are increasingly being utilized to distinguish individual sources of sound within a complex acoustic field. A non-traditional application of phased microphone arrays has recently been extended to the area of aircraft wake vortices. In particular, there is an ongoing effort to study the viability of using phased arrays to passively detect and track aircraft wake vortices ${ }^{1-6}$ near airports in order to increase safety as well as more efficiently utilize existing runways. Such arrays and the associated signal processing are designed on the basis of ray theory in an ambient medium. While this analysis approach may be adequate in many scenarios, it is not clear that it suffices when the propagation takes place through the atmosphere where strong winds and temperature gradients may be present. In this paper, a three-dimensional ray tracing code that can account for all atmospheric variability is developed. The code is then exercised to ascertain the effects of such variability on microphone array processing techniques.

\section{Analysis}

If $\mathbf{x}(t)$ is a point on the wavefront of an acoustic wave, then it will always lie on the wavefront if its velocity is given by Ref. 7

$$
\frac{d \mathbf{x}}{d t}=\mathbf{v}(\mathbf{x}, t)+\mathbf{n}(\mathbf{x}, t) c(\mathbf{x}, t)
$$

where $t$ is time, $\mathbf{n}(\mathbf{x}, t)$ is the unit normal to the wavefront with components $(l, m, n)$, and $\mathbf{v}(\mathbf{x}, t)$ and $c(\mathbf{x}, t)$ are the wind velocity and speed of sound, respectively, at the point $\mathbf{x}$ at time $t$. The change in the normal vector as the wavefront propagates is given by

Electrical Engineer, Advanced CNS Technologies Division, 55 Broadway.

$\dagger$ Acoustic Consultant, Titan Group, 300 Concord Road, Suite 400, Member AIAA.

* Aerospace Engineer, Advanced CNS Technologies Division, 55 Broadway, Senior Member AIAA. 


$$
\frac{d \mathbf{n}}{d t}=-[\nabla-\mathbf{n}\{\mathbf{n} \cdot \nabla\}] c-\sum_{k} n_{k}[\nabla-\mathbf{n}\{\mathbf{n} \cdot \nabla\}] v_{k}
$$

where $n_{k}$ and $v_{k}$ are the components of the normal and velocity vectors, respectively, and the $n_{k}$ are formally treated as constant in carrying out the differentiation.

It can readily be seen from Eq. (2) that, if there is no wind velocity and the speed of sound is constant $c(\mathbf{x}, t)=c_{0}$, then $\frac{d \mathbf{n}}{d t}=0$ and the direction of the normal never changes, i.e., $\mathbf{n}=\mathbf{n}_{0}$, a constant vector. Further,

Eq. (1) then reduces to $\frac{d \mathbf{x}}{d t}=\mathbf{n}_{0} c_{0}$ which shows that the point on the wavefront travels in the direction of the vector $\mathbf{n}_{0}$ at constant speed $c_{0}$ which is the assumption made in the phased array analysis. However, when there are winds and temperature gradients such that the speed of sound is not constant, the speed and direction of the propagation of sound may be altered. For this reason, Eqs. (1) and (2) have been programmed, and wind and temperature profile data collected from a test site have been employed to examine the real atmospheric effects.

\section{A. Code Validation}

For the purpose of code validation, the relation $\mathbf{n} \cdot \mathbf{n}=1$, required by the fact that $\mathbf{n}$ is a unit vector, was utilized. Another useful check on the programming may be developed when there is only one component of velocity, i.e., $v=w$ $=0$ and the velocity component and sound speed depend only upon $z$, i.e., $u=u(z)$ and $c=c(z)$. That is, the $x$ coordinate axis is placed in the direction of the prevailing wind. This is the so-called "stratified medium" assumption employed by Heath and McAninch. ${ }^{8}$ In that case, the third component of Eq. (1) and the first component of Eq. (2) become

$$
\begin{aligned}
& \frac{d z}{d t}=n c \\
& \text { and } \\
& \frac{d l}{d t}=\ln \left[\frac{d c}{d z}+l \frac{d u}{d z}\right]
\end{aligned}
$$

Combining these two equations yields

$$
\frac{d l}{d z}=\frac{l}{c}\left[\frac{d c}{d z}+l \frac{d u}{d z}\right]
$$

which is a nonlinear Bernoulli equation. Solving by standard techniques yields the solution

$$
l(z)=\frac{c(z)}{k-u(z)}
$$

where

$$
k=\frac{c\left(z_{0}\right)}{l\left(z_{0}\right)}+u\left(z_{0}\right)
$$

and $z_{0}$ is the $z$-coordinate of the initial starting position of the ray. Once $l(z)$ is known, a similar equation for $m(z)$, i.e.,

$$
\frac{d m}{d z}=\frac{1}{c}\left[\frac{d c}{d z}+l \frac{d u}{d z}\right] m
$$

may be derived and solved for 


$$
m(z)=\frac{m\left(z_{0}\right)}{l\left(z_{0}\right)} l(z)
$$

and finally $n(z)$ may be obtained from the relation

$$
n(z)= \pm \sqrt{1-l^{2}(z)-m^{2}(z)}
$$

Thus, the normal vector is known as a function of $z$ and the ray may be followed by merely integrating Eq. (1). This is equivalent to the "slowness vector" approach utilized by Heath and McAninch. ${ }^{8}$ These relations were also employed for code validation.

\section{B. Data Requirements}

The integration of the coupled set of first order ordinary differential equations given by (1) and (2) in full generality requires knowledge of all three wind velocity components $u, v$, and $w$ as well as the sound speed $c$ at all points in space that the ray passes through at the time at which it passes through. Note that these velocity components contain both the mean and turbulent parts of the atmospheric winds. The sound speed can be obtained from knowledge of the temperature via ideal gas assumption, which is sufficient for as long as periods of severe weather conditions are excluded. As a part of the overall wake acoustics study, extensive meteorological data have been collected at the Denver International Airport during summers of $2003^{2,6}$ and 2005. For the present paper, sample time-averaged data from the 2005 test will be used. The velocity measurements up to 106 feet above the ground are obtained from three three-component ultrasonic anemometers mounted on a meteorological tower. Three-component wind velocities from 200 feet to 2200 feet are obtained from a pulsed LIDAR operating in Velocity Azimuth Display (VAD) mode. A microwave radiometer is then used to provide the temperature profile from 0 to 600 meter height. Nevertheless, the time histories of the velocity components and sound speed at every point in the region of interest are not available. The measured profiles at the test site are thus assumed to be the same throughout the entire region of interest through which the rays would travel.

For example, Fig. 1 depicts the profile of the five-minute time average temperature over the range 0 to 2000 feet collected from Denver in 2005. The atmospheric mean temperature does not change rapidly compared to the time taken for an acoustic ray to traverse from the wake vortex source to the ground, especially if a concept of operation calls for tracking vortices acoustically within a nautical mile from the array. Further, the sound speed variation is only moderately sensitive to the temperature. Thus, it is believed that such short time average data will be sufficient for the studies envisioned.

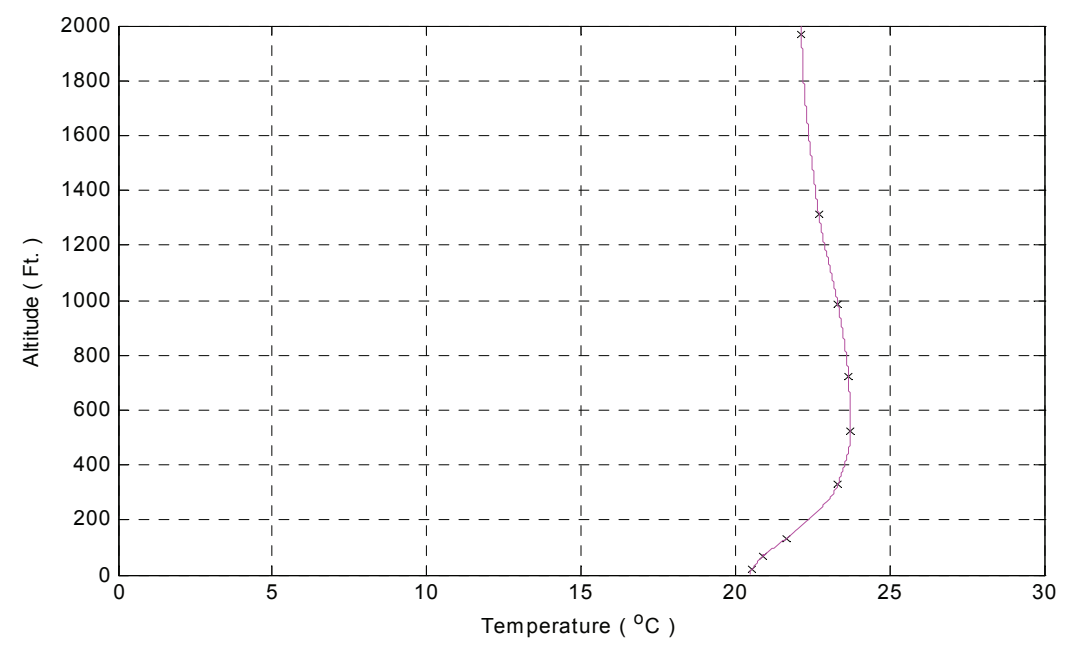

Figure 1. Time Average Temperature Profile.

Similarly, since the vertical (or $w$-component) of the wind vector in the planetary boundary layer tends to be small compared to the wind speed in the horizontal direction, it has been decided to initially ignore the vertical 
component in the ray tracing calculations. Fig. 2 displays the horizontal wind profile from the same five-minute time average period as in Fig. 1. Although the wind speed only varies from 0 to 12 knots over this range, the gradients of this wind speed can have important effects upon the acoustic rays. It is interesting to note that data from Fig. 1 indicate the existence of an inversion layer at approximately 500 feet altitude. The corresponding horizontal wind velocity below 500 feet, shown in Fig. 2, deviates from the standard logarithmic (or power-law) velocity profile.

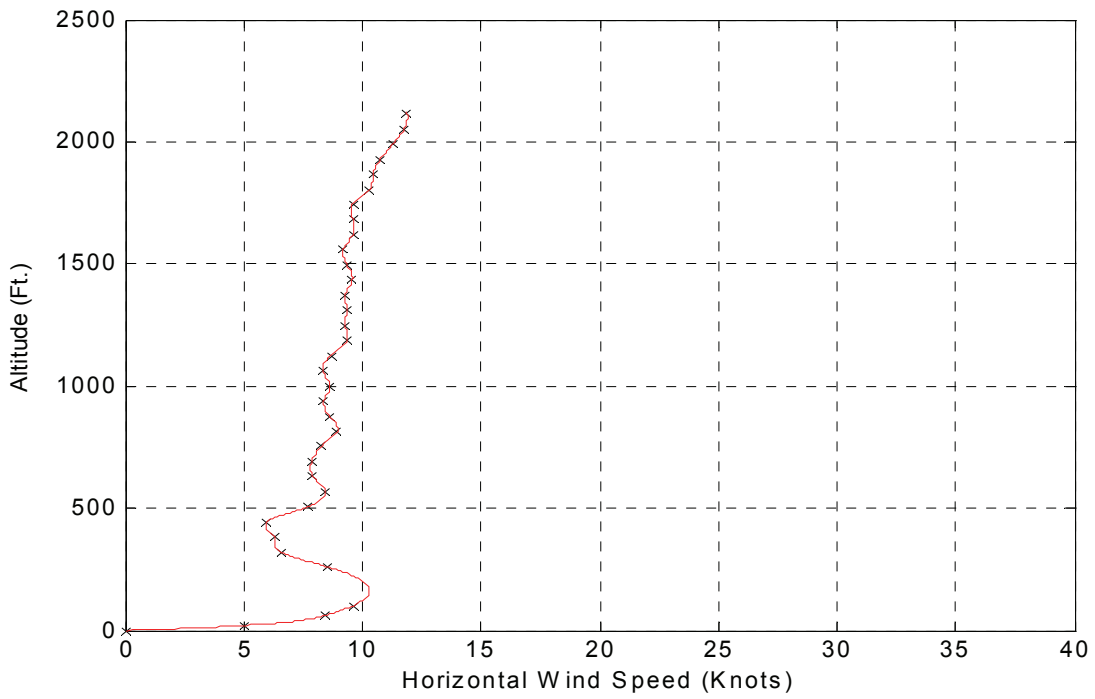

Figure 2. Horizontal Wind Speed Profile.

Fig. 3 displays the five-minute time average profile of the angle of the horizontal wind speed over the range 0 to 2200 feet. The angle is defined such that zero degree is a wind blowing towards (true) east and an increasing value represents a counterclockwise rotation in the wind vector. Note that this angle varies significantly over this range. Thus, the $u$ - and $v$-components of the wind velocity vector vary considerably with altitude. These variations will also have substantial effects upon the acoustic ray propagation. Although Figs. 2 and 3 again represent time average properties, the propagation studies will initially be carried out based upon such data. Turbulent velocity components can then easily be added in later if sensitivity studies deem them to be necessary.

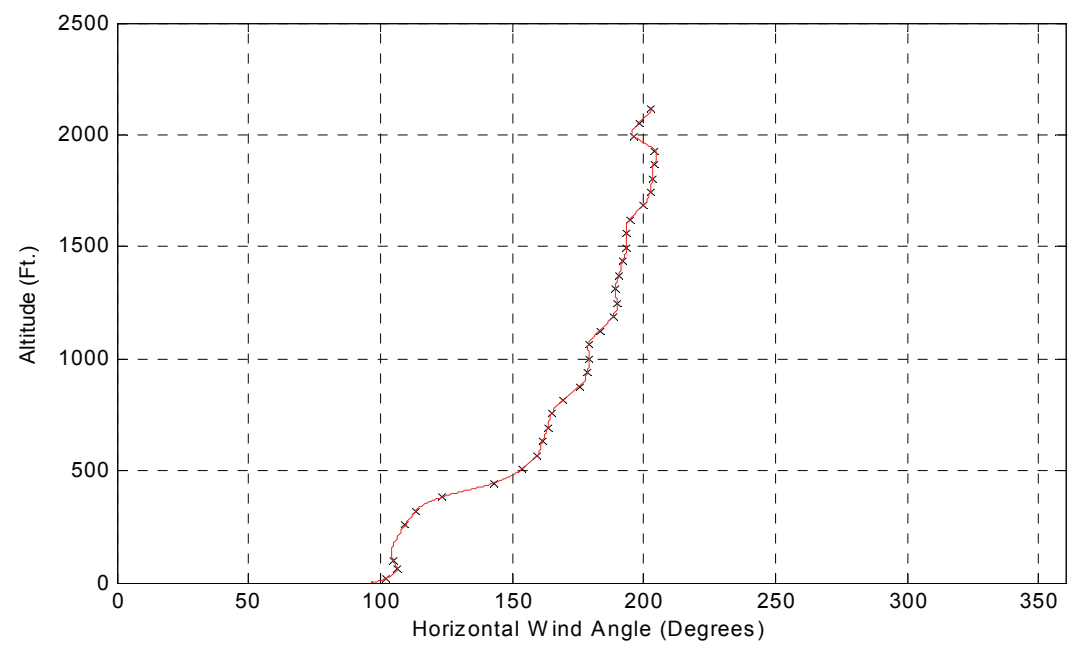

Figure 3. Angle of Horizontal Wind Speed Relative to East. 


\section{Applications to Acoustic Array Processing}

The above Eqs. (1) and (2) have been programmed in all generality in order to assess real atmospheric effects on the processing of acoustic array data. The approach employed is to examine how the travel times from the source to the microphones in the array are altered by propagation through the existing atmosphere as compared to those in an ambient atmosphere.

\section{A. Example}

As a trivial example of the analysis, the array employed by Alix et $\mathrm{al}^{3}$ for wake vortex sound measurement at Logan International Airport in Boston is examined. The linear array consisted of four microphones spaced 5.74 feet apart and focused on a point 42 feet high and 11 feet in front of the leading microphone. This array was parallel to the runway across which a steady wind component of $2.7 \mathrm{mph}$ (=3.96 feet per second) was blowing toward the microphones. The origin of coordinates is placed on the ground 11 feet in front of the leading microphone with the positive $\mathrm{x}$-axis running along the array. Thus, the microphone positions lie at $(11,0,0),(16.74,0,0),(22.48,0,0)$ and $(28.22,0,0)$ in feet. For simplicity, the sound speed is taken as constant equal to 1130 feet per second and the velocity vector is taken to have only one component, $v_{2}=-3.96$ feet per second, again constant.

In this case, Eq. (2) again reduces to $\frac{d \mathbf{n}}{d t}=0$ indicating that the direction of sound propagation does not change from its initial direction. However, letting $\mathbf{x}=(x, y, z)$, Eq. (1) becomes

$$
\begin{gathered}
\frac{d x}{d t}=n_{1} c_{0} \\
\frac{d y}{d t}=v_{2}+n_{2} c_{0} \\
\frac{d z}{d t}=n_{3} c_{0}
\end{gathered}
$$

which are readily integrated to yield

$$
\begin{gathered}
x(t)=n_{1} c_{0} t+x_{0} \\
y(t)=\left(v_{2}+n_{2} c_{0}\right) t+y_{0} \\
z(t)=n_{3} c_{0} t+z_{0}
\end{gathered}
$$

Thus a ray starting from the focus location, $\mathbf{x}_{0}=(0,0,42)$, toward one of the microphones, i.e. implying that $n_{2}=0$, would hit the ground at time $t=-\frac{z_{0}}{n_{3} c_{0}}$. It would have traveled a distance $x=n_{1} c_{0} t=-\frac{z_{0} n_{1}}{n_{3}}$ in the $x$-direction in that time. However, it would also have traveled a distance $y=v_{2} t=-\frac{z_{0} v_{2}}{n_{3} c_{0}}$ in that time. Thus, that ray will not strike the microphone at all.

The ray which will strike the microphone is one for which $v_{2}+n_{2} c_{0}=0$, i.e., one leaving the focus location in a direction such that $n_{2}=-\frac{v_{2}}{c_{o}}=M>0$ (where $M$ is the Mach number of the wind towards the microphones) since $v_{2}<0$. Because $\mathbf{n}$ is a unit vector, $n_{1}{ }^{2}+n_{3}{ }^{2}=n_{1}{ }^{2}+\frac{z_{0}{ }^{2}}{x_{m}{ }^{2}} n_{1}{ }^{2}=1-M^{2}$ where $x_{m}$ is the $x$ coordinate of the microphone. Thus, $n_{1}=\sqrt{\frac{1-M^{2}}{1+\left(z_{0} / x_{m}\right)^{2}}}$ and $n_{3}=-\sqrt{1-M^{2}-n_{1}^{2}}$ for the ray striking the microphone. From these relations, it can be shown that the time at which the ray will strike the microphone is increased by the factor $1 / \sqrt{1-M^{2}}$ for each of the microphones. For the simple example considered, $M=0.0035$. Thus, the factor of increase is only 1.000006125 , entirely negligible for frequencies below $2 \mathrm{kHz}$ in the array considered. As this was the 
cut-off frequency for the measurements, it can be seen that the array analysis performed by Alix et $\mathrm{al}^{3}$ was certainly adequate. However, under conditions of higher wind velocity, greater source/array distance or wind and temperature gradients, the more precise analysis proposed in this paper may be required.

\section{B. Analysis of Experimental Configuration at Denver International Airport}

The current study is prompted by a new experimental setup to examine detection and tracking aircraft wake vortex sound with a longer sensor stand-off distance at Denver International Airport. As mentioned previously, extensive wind and temperature profiles were collected there which are used as the meteorological input in the present studies. The following example is used for the purpose of investigating the potential atmospheric effects: a linear array of 20 microphones spaced $5 \mathrm{~m}$ apart was considered. The vortex sound is idealized as a point source and is originated from an altitude of $223 \mathrm{~m}$ at a lateral distance of $230 \mathrm{~m}$ from the 1st microphone in the array and $1000 \mathrm{~m}$ downstream. Fig. 4 displays an overhead view of the source and array geometry.

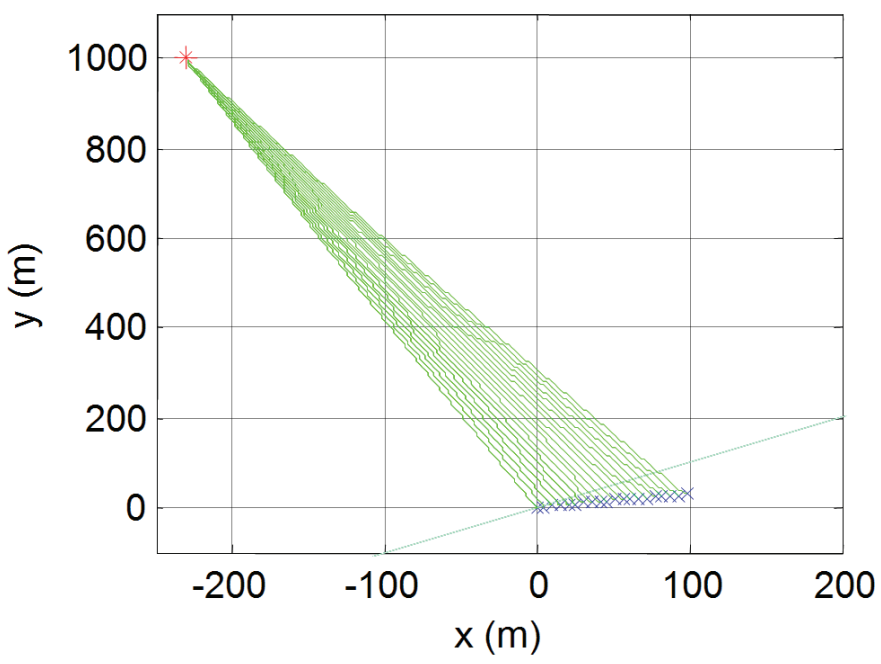

Figure 4. Overhead View of Source and Array Geometry.

The $y$-axis is aligned with the runway and aircraft land in the direction of decreasing $y$ values. The red point indicates the source, the blue dots are the microphones and the green rays are those which would be found in an ambient atmosphere. Note that the geometry of the aforementioned array is constructed for the purpose of the current example and does not represent details of a configuration under examination.

An iterative "shooting method" was coded which first propagated a ray in the direction of the direct path between the source and one of the microphones. Depending upon where this ray hit the ground, the code would automatically adjust the initial angle of the ray and compute a new ray path until the ray that strikes the microphone is determined. The difference between the two rays is not large, not even observable on a plot of the paths of the two rays over the entire range between source and receiver. To give some idea of the different paths, Figure 5 is a plot of the distance $d=\sqrt{(\Delta y)^{2}+(\Delta z)^{2}}$ between the two rays as a function of the $x$-coordinate. Note that this distance, which is of course zero at the source and receiver, never gets much larger than $15 \mathrm{~m}$. 


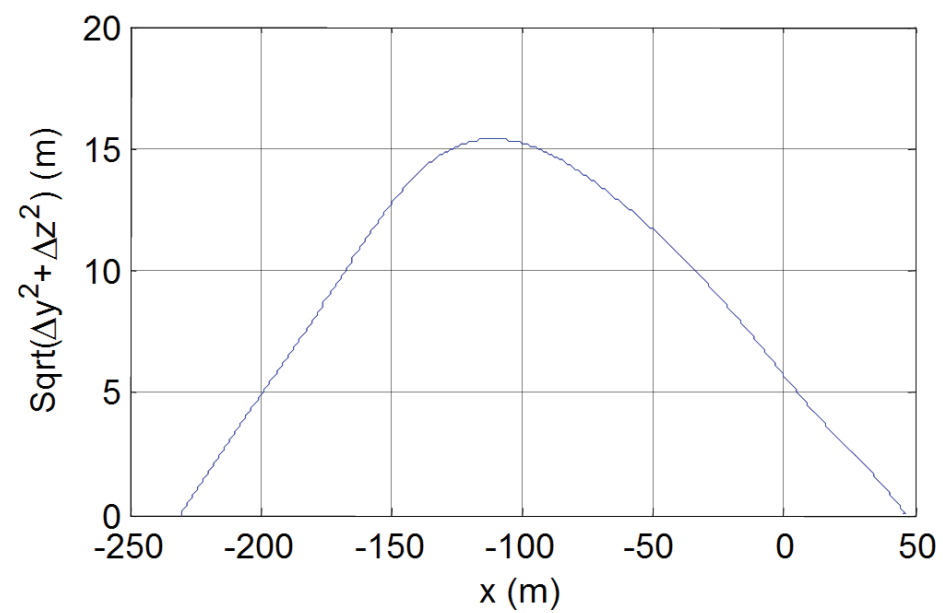

Figure 5. Distance between Real and Ambient Atmosphere Rays.

Nevertheless, the time taken by the two rays is significantly different. Fig. 6 depicts the time difference between the two rays, i.e., time taken by ray in real atmosphere minus time taken by ray in ambient atmosphere, as a function of microphone number (numbered sequentially starting with the 1 st microphone described above):

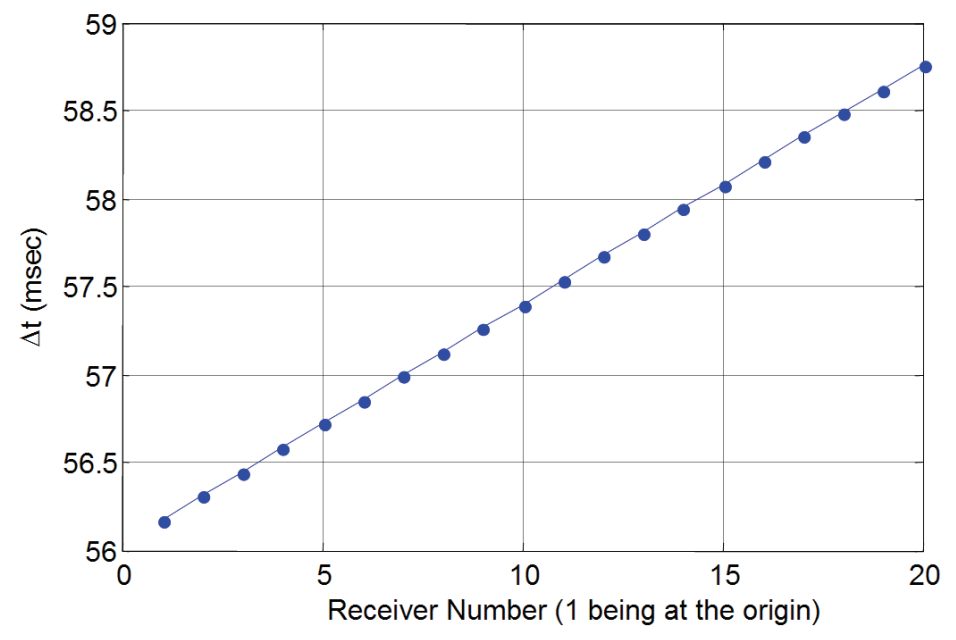

Figure 6. Travel Time Difference as Function of Microphone Number.

Note that this travel time difference increases with microphone number and is substantial, approximately $57 \mathrm{~ms}$. The reason for this is that the rays travel at different speeds. Fig. 7 displays the incremental distance traveled by the various rays in a time interval $\Delta t=10^{-4} \mathrm{sec}$ as a function of altitude: 


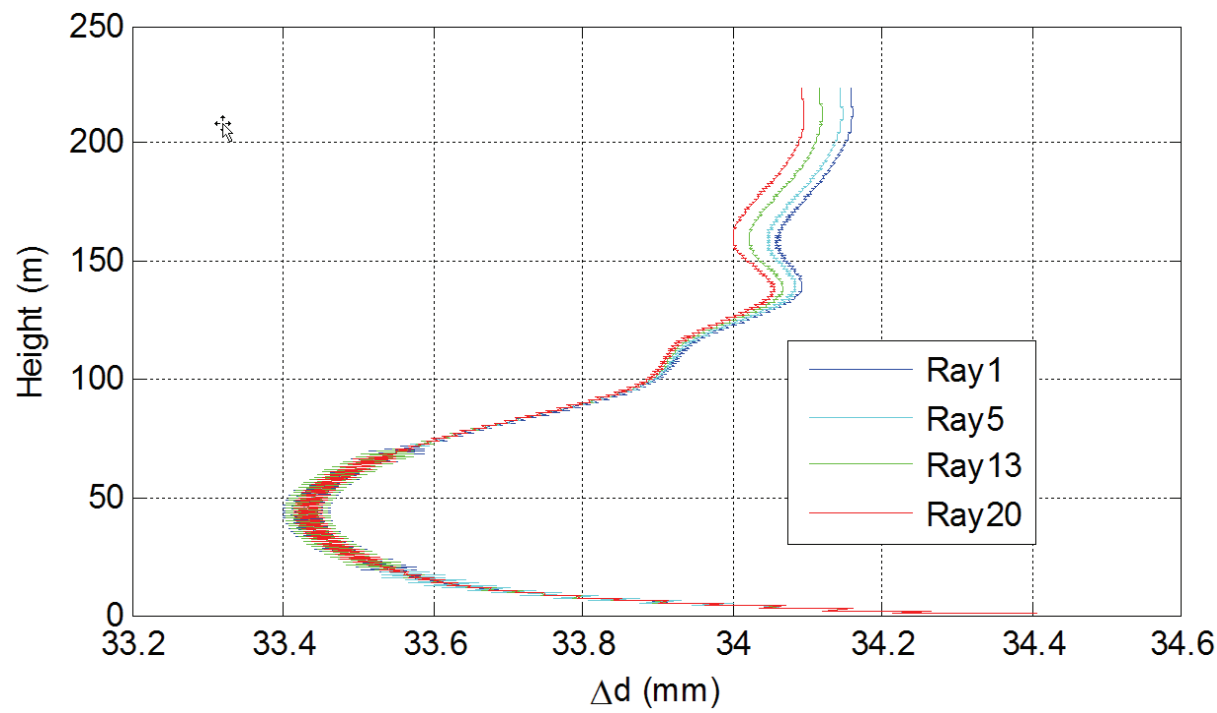

Figure 7. Incremental Travel Distance as Function of Altitude.

The ray in the ambient medium would, of course, travel the same distance in each time interval, near $34 \mathrm{~mm}$. Thus, it can be seen that over much of its path, the ray in the real atmosphere is traveling more slowly than would the ray in the ambient medium. However, it is not the magnitude of the time delay, but the differences in the delay between microphones that are important for the array analysis. The consequences of these observed delay differences on the design of the acoustic array are next examined.

The theory behind the design of acoustic arrays is that if the signals measured by all microphones in the array are summed up, then the aspects of the signal which are coherent in all the microphones will add constructively. The incoherent aspects will tend to average out to a lower signal level. Thus, an assumed source location or focal point is chosen and signal travel times from the focal point to the various microphones are calculated for propagation in an ambient atmosphere. This calculation yields the times by which the various signals must be delayed in order to add up properly. The performance of the array for signals from other source locations is degraded compared to those from the assumed location since the delay times are not quite correct. This degradation yields the beam width of the array that is a function of source frequency. Small time delay errors become more important at higher frequencies.

This design philosophy is adequate for propagation in the real atmosphere as long as the rays from the assumed source to the microphones in the array are equally delayed or accelerated by the atmospheric effects. In that case, the array design delay times are unaffected. Clearly, this fortunate circumstance becomes less likely for larger arrays or longer source/array propagation distances. From Fig. 6 it can be seen that, for the example considered here, there are propagation delay differences on the order of $2.5 \mathrm{msec}$. These differences occur over the $100 \mathrm{~m}$ extent of the array. Table 1 below depicts signal periods for frequencies of typical interest in wake vortex tracking. Note that $2.5 \mathrm{msec}$ is substantial compared to these periods, $T$. Fig. 6 suggests the time delay to scale linearly with array size. Taking an error of one third of a period as acceptable and assuming linear scaling, Table 1 also displays the maximum linear array size permitted at a few represented frequencies, beyond which the length of the array would be considered too large under the sample atmospheric condition considered in the paper.

Table 1. Maximum Array Size as Function of Frequency

\begin{tabular}{|c|c|c|c|}
\hline Freq $(\mathrm{hz})$ & $T(\mathrm{sec})$ & $T / 3(\mathrm{msec})$ & $d_{\max }(\mathrm{m})$ \\
\hline 100 & .01 & 3.33 & 133 \\
\hline 200 & .005 & 1.66 & 66.3 \\
\hline 300 & .00333 & 1.11 & 44.3 \\
\hline 400 & .0025 & .833 & 33.2 \\
\hline
\end{tabular}


Of course, a better way to handle such real atmospheric effects would be to incorporate them into the processing of the array data, i.e., to base the design of the array processing algorithm upon the delay times calculated by a threedimensional ray code using timely meteorological data. In that case, the full advantage of the array measurement technology could be preserved.

\section{Conclusions}

The paper presents an example to illustrate atmospheric propagation effects on time delay differences on which acoustic array designs are based. These effects are shown to be particularly important for large arrays and long propagation distances. There are two ways in which such effects can be ameliorated: One is to utilize a three dimensional ray tracing code with timely meteorological data to calculate these effects and incorporate them into the processing of the array data. The other is to limit the size of the array depending upon the maximum frequency of interest. The first of these approaches, although requiring more effort, is preferable in that it preserves the fidelity of the acoustic array.

\section{Acknowledgements}

The authors wish to express their appreciation to the NASA's Aeronautics Research Mission Directorate, and in particular the Airspace Systems Program, for funding the exploration of this fundamental mechanism for detecting aircraft wakes, and to the program manager, Wayne Bryant, for ensuring that the work focused on addressing the core issues. In addition, we thank Kevin Clark and Amir Tabrizi of USDOT Volpe Center, as well as Leo Jacobs of L3 Communications - Titan Group, for collecting and processing the meteorological data. Likewise, the overall guidance and encouragement from Michael Geyer of USDOT Volpe Center is acknowledged with appreciation.

\section{References}

${ }^{1}$ Michel, U., and Böhning, P., "Investigation of Aircraft Wake Vortices With Phased Microphone Arrays," AIAA Paper No. 2002-2501, 8th AIAA/CEAS Aeroacoustics Conference, Breckenridge, CO, June 2002.

${ }^{2}$ Dougherty, R. P., Wang, F. Y., Booth, E. R., Watts, M. E., Fenichel, N., and D'Errico, R. E., "Aircraft Wake Vortex Measurements at Denver International Airport," AIAA Paper No. 2004-2880, 10th AIAA/CEAS Aeroacoustics Conference, Manchester, UK, May 2004.

${ }^{3}$ Alix, D. C., Simich, P. D., Wassaf, H., and Wang, F. Y., "Acoustic Characterization of Wake Vortices in Ground Effect," AIAA Paper No. 2005-0260, 43rd AIAA Aerospace Sciences Meeting and Exhibit, Reno, NV, January 2005.

${ }^{4}$ Booth, E. R., Jr., and Humphreys, W. M., Jr., "Tracking and Characterization of Aircraft Wakes Using Acoustic and Lidar Measurements," AIAA Paper No. 2005-2964, 11th AIAA/CEAS Aeroacoustics Conference, Monterey, CA, May 2005.

${ }^{5}$ Fine, N. E., and Kring, D. C., "Opto-Acoustic Tracking of Aircraft Wake Vortices," AIAA Paper No. 20052965, 11th AIAA/CEAS Aeroacoustics Conference, Monterey, CA, May 2005.

${ }^{6}$ Wang, F. Y., Wassaf, H., Gulsrud, A., Delisi, D. P., and Rudis, R. P., "Acoustic Imaging of Aircraft Wake Vortex Dynamics," AIAA Paper No. 2005-4849, 23rd AIAA Applied Aerodynamics Conference, Toronto, Canada, June 2005.

${ }^{7}$ Pierce, A. D., Acoustics: An Introduction to Its Physical Principles and Applications, Acoustical Society of America, Second Printing, 1991, Chapter 8.

${ }^{8}$ Heath, S. L., and McAninch, G. L., "Propagation Effects of Wind and Temperature on Acoustic Ground Contour Levels," AIAA Paper No. 2006-0411, 44th AIAA Aerospace Sciences Meeting and Exhibit, Reno, NV, January 2006. 